\title{
Differential associations of early callous-unemotional, oppositional, and ADHD behaviors: multiple domains within early-starting conduct problems?
}

\author{
Rebecca Waller, ${ }^{1}$ Luke W. Hyde, ${ }^{1,2,3}$ Adam S. Grabell, ${ }^{4}$ Martha L. Alves, ${ }^{1}$ and \\ Sheryl L. Olson ${ }^{1,2}$ \\ ${ }^{1}$ Department of Psychology, University of Michigan, Ann Arbor, MI; ${ }^{2}$ Center for Human Growth and Development, \\ University of Michigan, Ann Arbor, MI; ${ }^{3}$ Institute for Social Research, University of Michigan, Ann Arbor, MI; \\ ${ }^{4}$ Department of Psychology, University of Pittsburgh, Pittsburgh, PA, USA
}

\begin{abstract}
Background: Early-starting child conduct problems (CP) are linked to the development of persistent antisocial behavior. Researchers have theorized multiple pathways to $\mathrm{CP}$ and that $\mathrm{CP}$ comprise separable domains, marked by callous-unemotional (CU) behavior, oppositional behavior, or ADHD symptoms. However, a lack of empirical evidence exists from studies that have examined whether there are unique correlates of these domains. Methods: We examined differential correlates of CU, oppositional, and ADHD behaviors during the preschool years to test their potentially distinct nomological networks. Multimethod data, including parent and teacher reports and observations of child behavior, were drawn from a prospective, longitudinal study of children assessed at age 3 and age $6(N=240$; $48 \%$ female). Results: Dimensions of CU, oppositional, and ADHD behaviors were separable within Confirmatory Factor Analyses across mother and father reports. There were differential associations between CU, oppositional, and ADHD behaviors and socioemotional, cognitive, and behavioral outcomes: CU behavior was uniquely related to lower moral regulation, guilt, and empathy. ADHD was uniquely related to lower attentional focusing and observed effortful control. Finally, CU behavior uniquely predicted increases in teacher-reported externalizing from ages 3-6 over and above covariates, and ADHD and oppositional behavior. Conclusions: Consistent with theory, dimensions of CU, ADHD, and oppositional behavior demonstrated separable nomological networks representing separable facets within early-starting CP. Keywords: Callous-unemotional, conduct problems, oppositional, attention deficit/ hyperactive, nomological network.
\end{abstract}

\section{Introduction}

Early and persistent conduct problems (CP) undermine family well-being and represent the primary reason for youth referrals to clinicians (Kazdin, Whitley, \& Marciano, 2006). A consistent predictor of adolescent and adult antisocial behavior is childhood CP that may start as early as age 3 (Shaw \& Gross, 2008). Early-starting CP also put children at risk for developing other mental health problems, including substance use and depression (Odgers et al., 2008). However, heterogeneity in childhood CP has led to numerous subgrouping approaches, each potentially associated with distinct etiologies with implications for basic research and treatment. For example, three pathways to childhood $\mathrm{CP}$ have been proposed, differentiated by high emotional dysregulation (oppositional behavior), lack of inhibition and impulsivity (ADHD symptomatology), and emotional hyporesponsivity and conscience deficits [callousunemotional (CU) behavior; Frick \& Morris, 2004]. In the current study, we sought to test whether these separable facets within early-starting $\mathrm{CP}$ (i.e. CU vs. ADHD vs. oppositional behaviors) were associated with distinct nomological networks.

Conflict of interest statement: No conflicts declared.
Distinguishing between oppositional, ADHD, and $\mathrm{CU}$ behavior has intuitive and theoretical appeal, not least because of the implications for tailoring interventions to match children's characteristics. Children with oppositional behavior exhibit negative emotionality, elevated internalizing symptoms, and difficulties regulating anger (Stringaris \& Goodman, 2009), representing one potential 'hot' pathway to $\mathrm{CP}$, characterized by hostile attributions and reactive aggression. In a second 'hot' pathway, children exhibit high levels of ADHD behavior, including poor inhibitory and attentional control and impulsivity (von Stauffenberg \& Campbell, 2007). Finally, a theorized 'cold' pathway to $\mathrm{CP}$ is associated with $\mathrm{CU}$ behavior, characterized by hyporeactivity to affective cues, proactive aggression, and deficits in conscience (Blair, 2013; Frick, Ray, Thornton, \& Kahn, 2014). However, while theoretically appealing, a paucity of evidence exists from studies that have differentiated between these dimensions at very young ages or that have demonstrated specificity of correlates. The focus of the current study was thus to examine dimensions of ADHD, oppositional, and CU behavior and test whether they exhibited unique correlates.

An important first step is to demonstrate these behaviors are separable within measurement frameworks. In general, though highly correlated, $\mathrm{CU}$ behavior has emerged as distinct from ADHD and 
oppositional behaviors among older samples (Frick et al., 2014). However, older children rarely display $\mathrm{CP}$ if they have not already demonstrated behavior problems during preschool (Shaw, Gilliom, \& Giovannelli, 2000). Indeed, the preschool years represent an important developmental transition during which to examine these behaviors, as in this period, children are difficult to manage and undergo rapid psychological and physical changes (Shaw \& Gross, 2008). An overlapping literature has highlighted that individual differences in behaviors related to $\mathrm{CU}$ behavior, including conscience (Kochanska, 1997), also emerge during this period. Thus, studies are needed that examine the validity of distinguishing between ADHD, oppositional, and CU behaviors in very early childhood as it is likely to be a critical period for their development and for informing personalized intervention strategies.

In support of the distinction at very young ages, Willoughby and colleagues have demonstrated that 17 items of the preschool CBCL formed separable ADHD, oppositional, and CU behavior scales at age 3 (Willoughby, Mills-Koonce, Gottfredson, \& Wagner, 2014; Willoughby, Waschbusch, Moore, \& Propper, 2011). Studies have also shown that ADHD, oppositional, and CU symptom factors derived from other common behavior rating scales differentially predict outcomes in preschool samples (e.g. Hyde et al., 2013). Our preliminary study aim was to provide a further test of the three-factor model proposed by Willoughby and colleagues to determine whether ADHD, oppositional, and CU behaviors can be measured as separable constructs at age 3 .

Beyond measurement however, a novel empirical question surrounds construct validity and whether dimensions of ADHD, oppositional, or CU behavior exhibit distinct socioemotional, behavioral, or cognitive correlates. Willoughby et al. (2011) provided initial support for the distinction among 3-year olds $(N=178)$. Children with high-oppositional/CU behavior showed low fear and reduced responsivity compared to children with high oppositional-only, although the small subgroups ( $\mathrm{ns}=7-12$ ) makes it difficult to generalize these findings. Subsequently, Willoughby et al. (2014; $N=1176$ ) found that a high CU behavior subgroup of 3-year olds had higher teacher-rated aggression over time. However, creation of subgroups renders some loss of power even with the larger sample size, and limits the conclusions that can be drawn about three potential domains of early-starting CP.

Indeed, no previous studies have examined unique correlates of ADHD, oppositional, and CU behavior scales within a dimensional framework, taking advantage of multiple informants and settings, and employing observations of child behavior to test potentially distinct nomological networks. Thus, in the current study we examined differential associations with theoretically relevant constructs, controlling for overlap between dimensions. Specifically, we tested the hypothesis that ADHD, oppositional, and
CU behaviors exhibit specific correlates indexed via unique associations with measures of emotion regulation, effortful control, conscience, Theory of Mind (ToM), and emotion understanding. Thus, we aimed to provide a thorough examination of the potentially distinct nomological networks of these dimensions at a very young age. Indeed, while the notion that dimensions of ADHD, oppositional, and CU behavior exhibit differential correlates is appealing, very little research has addressed this assumption empirically. Our sample included children from a prospective longitudinal study, oversampled for externalizing behaviors, with rich parent-reported, teacherreported, and observational measures, providing a range in early-starting CP and sophisticated measurement of core constructs.

First, we assessed parent-reported anger/frustration and hypothesized that this would be uniquely associated with oppositional behavior once overlap in ADHD and CU behavior was accounted for, representing a 'hot' domain of early-starting CP characterized by irritability and difficulties in regulating negative emotionality. We also examined characteristics relevant to ADHD and a second potential 'hot' domain of CP. Specifically, we hypothesized that lower parent-reported attentional focus and lower observed effortful control would be uniquely related to ADHD behavior, consistent with deficient behavioral inhibition and attention representing central diagnostic features of ADHD (e.g. Barkley, 1997). Third, we hypothesized that CU behavior would be uniquely associated with lower parent-reported fear, deficits in conscience, and escalation of behavior problems, consistent with a theorized 'cold' CP domain (Frick \& Morris, 2004). Specifically, we examined whether $\mathrm{CU}$ behavior was uniquely related to poorer moral development, lower empathy, and lower guilt (Blair, 2013; Frick et al., 2014). We also examined whether $\mathrm{CU}$ behavior uniquely predicted increases in teacher-reported externalizing behavior from ages 3-6 (i.e. across informants and settings). In particular, we wanted to examine whether assessing CU behavior at age 3 adds to the predictive validity of early school-aged behavior problems beyond simply assessing existing behavior problems.

Fourth, we assessed whether ToM differed across dimensions. ToM represents a salient and theoretically relevant target of investigation in relation to $\mathrm{CU}$ behavior. In particular, deficits in cognitive empathy appear central to autism spectrum disorders (BaronCohen \& Wheelwright, 2004) and it is possible that deficits in empathy among high CU youth could also derive from more basic deficits in cognitive processes, such as ToM. Alternatively, CU behavior may be related specifically to affective components of empathy. While of potential theoretical importance, only one previous study has assessed ToM and CU behavior among youth, reporting no significant association (Jones, Happé, Gilbert, Burnett, \& Viding, 2010). In the current study, we thus examined 
different components of empathy separately to identify specific deficits related to $\mathrm{CU}$ behavior. We separated a cognitive understanding of others' thoughts and beliefs (ToM) from an observational assessment of others' emotions. We hypothesized that in addition to deficits in empathic concern, CU behavior would also be uniquely related to a compromised understanding of emotions, but intact ToM. Finally, we hypothesized that CU behavior at age 3 would uniquely predict increases in teacherreported externalizing behavior problems and higher levels of reactive and proactive aggression at age 6 .

\section{Methods}

\section{Participants}

Participants were 240 children (118 girls) and their parents who are part of an ongoing longitudinal study of young children at risk for CP (Olson, Sameroff, Kerr, Lopez, \& Wellman, 2005). Children were 3 years old at Time 1 (T1; $M=41.41, S D=2.09$ months) and 6 years old at Time 2 (T2; $M=68.87, S D=3.84$ months). Families were recruited from preschools, newspaper advertisements, and referrals. Screening questionnaires and telephone interviews were used to determine appropriateness for participation and to obtain consent. There was intentional oversampling of children in the upper range of the Externalizing Problems scale from the Child Behavior Checklist/2-3 (Achenbach, 1992). Most children were of European American heritage (86\%), with others self-identifying as African American (5\%) or biracial (8\%). Most mothers were married (89\%), 3\% lived with a partner, $5 \%$ were single, and 3\% were divorced. Median annual family income was $\$ 52,000$ (range, $\$ 20,000-\$ 100,000$ ). Retention from T1T2 was high $(88 \%)$ and families who dropped out did not differ on any comparisons of study variables or sociodemographic variables (Olson, Lopez-Duran, Lunkenheimer, Chang, \& Sameroff, 2011).

\section{Procedures}

Mothers $(n=240)$ and fathers $(n=145)$ completed questionnaires in their homes, and were given $\$ 100$ for participation. Participating teachers also completed questionnaires and were given gift certificates. At T1, children $(n=227)$ participated in a 4-hr laboratory session involving a series of cognitive and self-regulatory tasks (Kerr, Lopez, Olson, \& Sameroff, 2004).

\section{Measures}

Covariates. Parents answered questions relating to child gender, age, and family income. Children's cognitive functioning was assessed using the Vocabulary subtest of Wechsler's Preschool and Primary Scale of Intelligence-Revised (Wechsler, 1989).

Child behavior problems (parent-reported and teacher-reported). At T1, mothers and fathers completed the CBCL/2-3 (Achenbach, 1992), a 99-item measure of behavioral and emotional problems. Consistent with previous studies (Willoughby et al., 2011), we selected 17 items that assessed a five-item CU scale (e.g. 'lack of guilt after misbehavior'), a six-item ADHD scale (e.g. 'can't stand to wait'), and a six-item oppositional scale (e.g. 'defiant'). At T1 and T2, teachers completed the Caregiver/Teacher Report Form, Ages
2-5 (Achenbach, 1997). We used the broadband externalizing behavior subscale, which exhibited high internal consistency (T1, $\alpha=.96 ; \mathrm{T} 2, \alpha=.94)$. At T2, teachers also completed the Inventory of Peer Relations (Dodge $\&$ Coie, 1987). This 20-item scale comprises measures of reactive $(\alpha=.87$; 'when teased, strikes back') and proactive ( $\alpha=.85$; 'bullies others') aggression, which have been used previously in this sample (Olson et al., 2011).

Child temperament (parent-reported). At T1, parents completed an abbreviated 195-item version of Rothbart's Child Behavior Questionnaire (CBQ; Ahadi, Rothbart, \& Ye, 1993) to assess child temperament. We used three CBQ scales: Attentional Focusing $(\alpha=.85$; e.g. 'when picking up toys, keeps at task until done'); Fear ( $\alpha=.73$; e.g. 'is not afraid of large dogs'); and Anger/Frustration ( $\alpha=.77$; e.g. 'temper tantrums'; Olson et al., 2011).

Child conscience (parent-reported). At T1, parents completed the 'My Child' questionnaire (Kochanska, DeVet, Goldman, Murray, \& Putnam, 1994), a 100-item assessment of early conscience development with demonstrated reliability and validity among samples of young children (Kochanska et al., 1994). We used two composite scales and one subscale: Moral Regulation (composite of Internalized Conduct, Apology, and Confession subscales; $\alpha=.90$, e.g. 'tells parents after wrongdoing'), Guilt (composite of Guilt and Concern for Good Feeling with Parent subscales; $\alpha=.71$, e.g. 'feels bad when reminded about wrongdoing'), and the Empathy subscale $(\alpha=.89$, e.g. 'asks, "what's wrong?" when seeing someone in distress'; Kerr et al., 2004; Kochanska et al., 1994).

Child ToM (observed). At T1, we assessed ToM using the 'False Belief Prediction and Explanation Tasks-Revised' (Bartsch \& Wellman, 1989). Two tasks examined children's prediction and explanation of the choices of hypothetical protagonists who received erroneous information about the location of objects after locations were switched. Children had to predict where the protagonist would look for objects (prediction) and explain why the protagonist searched incorrectly (explanation). ToM total scores were computed by summing correct predictions and explanations. Scoring reliability (based on a random 15 children) was 97\%. Disagreements were settled through consultation with a team leader, an expert in ToM assessment (i.e. Henry Wellman). Reliability for ToM scores was good $(\alpha=.71$; Olson et al., 2011).

Child effortful control (observed). At T1, children completed six tasks from Kochanska, Murray, Jacques, Koenig, and Vandegeest's (1996) toddler-aged battery (turtle/ rabbit, whisper, tongue, tower, lab gift, and delay). Fifteen tests were videotaped and independently scored. Reliability was excellent (mean kappa $=.95$, range $=.92-98$; see Kochanska et al., 1996; Olson et al., 2005). As recommended by Kochanska et al. (1996), total observed effortful control scores were computed by summing individual subtest scores $(\alpha=.70$; Olson et al., 2005).

Child emotion understanding (observed). At T1, emotion understanding was assessed via three tasks that used vignettes enacted with a puppet (Denham, 1986). Children had to identify one of four emotions, predict the emotion that the puppet would express, and demonstrate understanding that the puppet could express a different emotion to that felt by the child. Children received two points for correctly identifying emotions, one point for recognizing emotions as good/bad, and zero points for incorrect responses/no response. Following 
Denham (1986), a composite emotion understanding score was created by summing scores across the three vignettes $(\alpha=.70)$. Based on a random 15 protocols, reliability of scoring was 100\% (Lane, Wellman, Olson, LaBounty, \& Kerr, 2010).

\section{Analytic strategy}

Differentiation of $C U, A D H D$, and oppositional behavior. We used Confirmatory Factor Analysis (CFA) in Mplus version 5.21 (Muthén \& Muthén, 1998-2007) to compare model fit for a one-factor model, three two-factor models, and a three-factor model. Models were estimated with mean and variance adjusted weighted least squares estimation (WLSMV), appropriate for ordinal items. Model fit was considered adequate if the Root Mean Square Error of Approximation (RMSEA) and Comparative Fit Index (CFI) values met established guidelines (RMSEA < .06 and CFI > .95; Hu \& Bentler, 1999). We tested models using mother and father reports to enable corroboration of fit within our sample. We carried out corrected chi-square differences test with DIFFTEST to test significant improvements in model fit.

Differential correlates of $C U, A D H D$, and oppositional behavior. We computed zero-order correlations between variables, including observational measures, parentreported T1 measures, and teacher-reported T1 and T2 measures. Next, we examined unique associations between $\mathrm{CU}$, ADHD, and oppositional behavior and outcomes using multiple regression and controlling for child gender, age, family income, and verbal ability in all models. We ran regression models with a full information maximum likelihood (FIML) approach, which accommodates missing data and provides less biased estimates than listwise or pairwise deletion (Schafer \& Graham, 2002), resulting in an effective sample size of 238 (covariance coverage: mother-reported data $=.98-.99$; observed $=.93-.96$; teacherreported $=.64-.79$ ).

\section{Results \\ Differentiation of $C U, A D H D$, and oppositional behavior}

Descriptive statistics are presented in Table 1. Consistent with our hypothesis, we found differentiation of CU, ADHD, and oppositional behaviors with the three-factor model providing the best fit. Good fit was replicated across mother and father reports. Corrected chi-square differences test using DIFFTEST indicated that the three-factor model provided significantly better fit than competing models (see Table S3). Factor loadings were moderate and significant (see Figure 1). The model fit equally well using father reports (see Table $\mathrm{S} 4^{1}$ ). Consistent with previous findings, latent correlations between factors were moderate-high (range, $r=.69-.77, p<.001$ ), indicating distinct but overlapping constructs. Finally, oppositional and ADHD subscales had good internal consistency (ADHD, $\alpha=.82$; oppositional, $\alpha=.85$ ), but the CU behavior subscale reliability did not fall within the acceptable range, $(\alpha=.59)$. However, our estimate is comparable with alphas reported for 3year olds using the same five $\mathrm{CU}$ behavior items $(\alpha=.65$, Willoughby et al., 2011; $\alpha=.55$, Willoughby et al., 2014). The lower alpha for CU behavior scales may stem from the few number of items $(n=5)$ and low alpha per se is not always indicative of the usefulness of a measure (Schmitt, 1996). However, aspects of behavior relating to $\mathrm{CU}$ behavior may also not be fully developed by age 3 (e.g. empathic concern), which should be considered alongside the findings (Hyde et al., 2013).

\section{Differential correlates of $C U, A D H D$, and oppositional behavior}

Zero-order associations. In zero-order correlations, ADHD, oppositional, and CU behavior were associated with lower attentional focusing and higher anger/frustration. Oppositional behavior was associated with higher levels of fear. ADHD, oppositional, and $\mathrm{CU}$ behaviors were all related to lower moral regulation, guilt, and empathy (Table 1). Similar zero-order correlations emerged when associations were examined across informant using father-reported outcomes (Table S2). In addition, in zero-order correlations, both ADHD and CU behaviors were associated with lower observed effortful control, ToM, and emotion understanding (Table 1). Finally, both ADHD and CU behaviors were related to higher teacher-reported externalizing behavior at $\mathrm{T} 1$ and $\mathrm{T} 2$, and higher reactive and proactive aggression at $\mathrm{T} 2$.

Unique associations (multiple regression). Next, we examined unique effects using multiple regression and tested whether CU, ADHD, and oppositional $^{2}$ behaviors were differentially related to outcomes, controlling for their overlap and for gender, age, family income, and verbal IQ (Table 2). ADHD behavior was uniquely associated with lower observed effortful control and lower mother-reported attentional focus. ADHD and oppositional behavior were associated with more anger/frustration. CU behavior was uniquely related to lower levels of mother-reported moral regulation, guilt, and empathy. Finally, ADHD behavior was related to lower observed emotion understanding, but there were no unique associations between any of the behavior dimensions and ToM. The pattern of findings was similar when associations were examined across informant (see Table S4 for father-reported outcomes). ${ }^{3}$

Finally, we examined prediction of T2 teacherreported externalizing behavior. All models controlled for covariates as before and included $\mathrm{T} 1$ teacher-reported externalizing behavior. Thus, we tested which of the three parent-reported behavior scales predicted increases in behavior problems over time and across both informant and setting (home vs. school). Controlling for overlap between subscales, only $\mathrm{CU}$ behavior predicted increases in teacher-reported externalizing behavior and more proactive aggression at T2 (Table 2). 


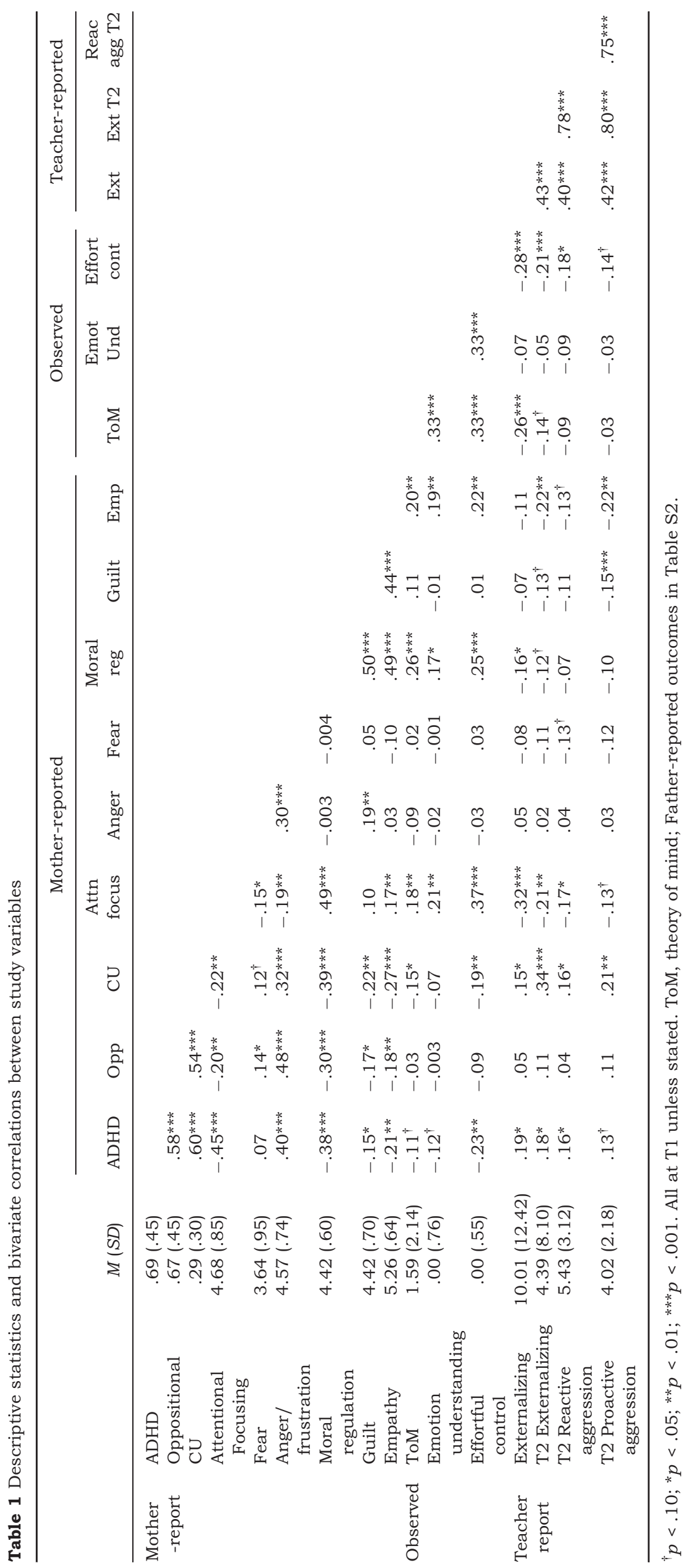




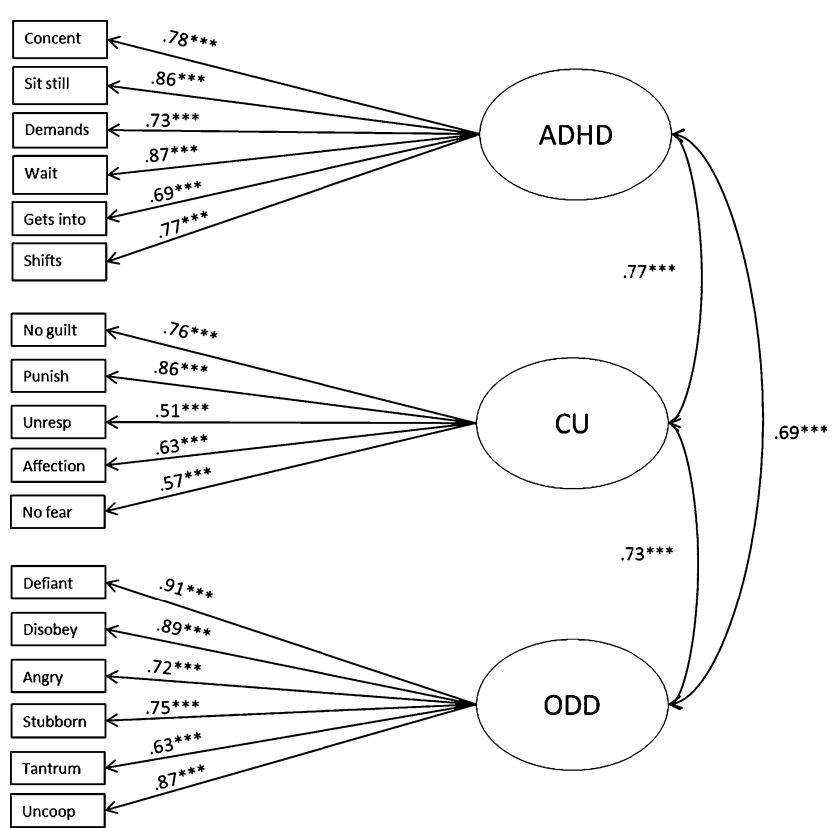

Figure 1 Factor structure and factor loadings of a three-correlated model differentiating between mother-reported CU, ADHD, and oppositional behaviors using $\mathrm{CBCL}$ items. $* * * p<.001$. $\chi^{2}(\mathrm{df})=256.57(116), p<.001 ; \mathrm{CFI}=.95 ;$ RMSEA $=.07$

\section{Discussion}

The current study provides further support for a three-factor model that distinguishes between ADHD, oppositional and $\mathrm{CU}$ behavior at age 3 (Willoughby et al., 2011). Beyond replicating this structure, the current study adds to the literature by demonstrating unique correlates of ADHD, oppositional, and CU behavior among very young children. Thus, our results provide support for the hypothesis that there are multiple and separable domains within early-starting CP (Frick \& Morris, 2004; Pardini \& Frick, 2013). In particular, we found empirical support for the assumption that three different behavior dimensions of ADHD, oppositional, and $\mathrm{CU}$ behavior are associated with unique nomological networks (see Figure 2).

First, consistent with our hypothesis, ADHD, and oppositional behaviors appeared to index 'hot' CP domains. Both ADHD and oppositional behavior were related to higher parent-reported anger/frustration. In addition, ADHD was related to lower observed effortful control and lower parent-reported attentional focus. Second, also consistent with our hypothesis, CU behavior was robustly and uniquely associated with lower moral regulation, guilt, and empathy across mother and father reports. Further, $\mathrm{CU}$ behavior was uniquely associated with increases in teacher-reported externalizing behavior and proactive aggression over time. Thus, our results support the notion that $\mathrm{CU}$ behavior, even in the preschool years designates children at risk for more severe and proactive antisocial behavior (Hyde et al., 2013) and appears distinguishable from other disruptive behaviors by specific deficits in conscience.
Further, CU behavior predicted increases in externalizing behavior over and above low empathy (see Table 2), suggesting that while CU behavior and empathy are related, they do not simply represent 'opposite sides of the same coin'. However, future studies are needed to examine the extent to which measurement of CU behavior and empathy overlap across different samples. Nevertheless, the prospective prediction of teacher-reported behavior problem underscores the value of measuring $\mathrm{CU}$ behavior in children as young as 3 years old. In particular, these findings highlight the utility of early prevention and intervention efforts incorporating CU behavior measures for use with preschool children, to identify those at the highest risk of future behavior problems.

Finally, although there were significant associations between $\mathrm{CU}$ and ADHD behavior and lower ToM and emotional understanding in zero-order correlations, in regression analyses controlling for overlap between subscales, only ADHD was related to lower emotion understanding. Our results are thus somewhat consistent with a previous study that reported no association between ToM and CU behavior (Jones et al., 2010), although the current study is novel in addressing this question in preschool-aged children. Thus, our findings suggest that despite deficits in conscience and empathy, high CU behavior appears related to intact cognitive understanding of others' emotions. Nevertheless, despite intact cognitive understanding, children with high $\mathrm{CU}$ behavior appear to lack emotional empathy, such as shared affect or feeling reciprocal emotion induced by another person's situation (Table 2; Blair, 2005). The finding that ADHD behavior was associated with lower emotion understanding is consistent with previous reviews suggesting ADHD is related to poorer emotion recognition skills, which may represent an early risk factor for peer rejection (Barkley, 1997).

There were a number of strengths to this study, including the prospective, longitudinal design, reports of child behavior from multiple informants and settings, and use of well-validated behavioral observations. Nevertheless, our results should be considered alongside several limitations. First, participants were drawn from a community sample comprised mostly of middle-class, Caucasian, and intact families. Thus, although this sample complements our previous work among high-risk samples (e.g. Hyde et al., 2013), results may not generalize to low-income families, families composed of ethnic minorities, or families with less stable structures. Second, shared method variance may have inflated associations between the scales, although the similar associations we found across informants somewhat allays this potential concern. Third, it should be noted that the CBCL was not developed to provide a nuanced assessment of the behavior dimensions assessed in the current study, particularly CU 


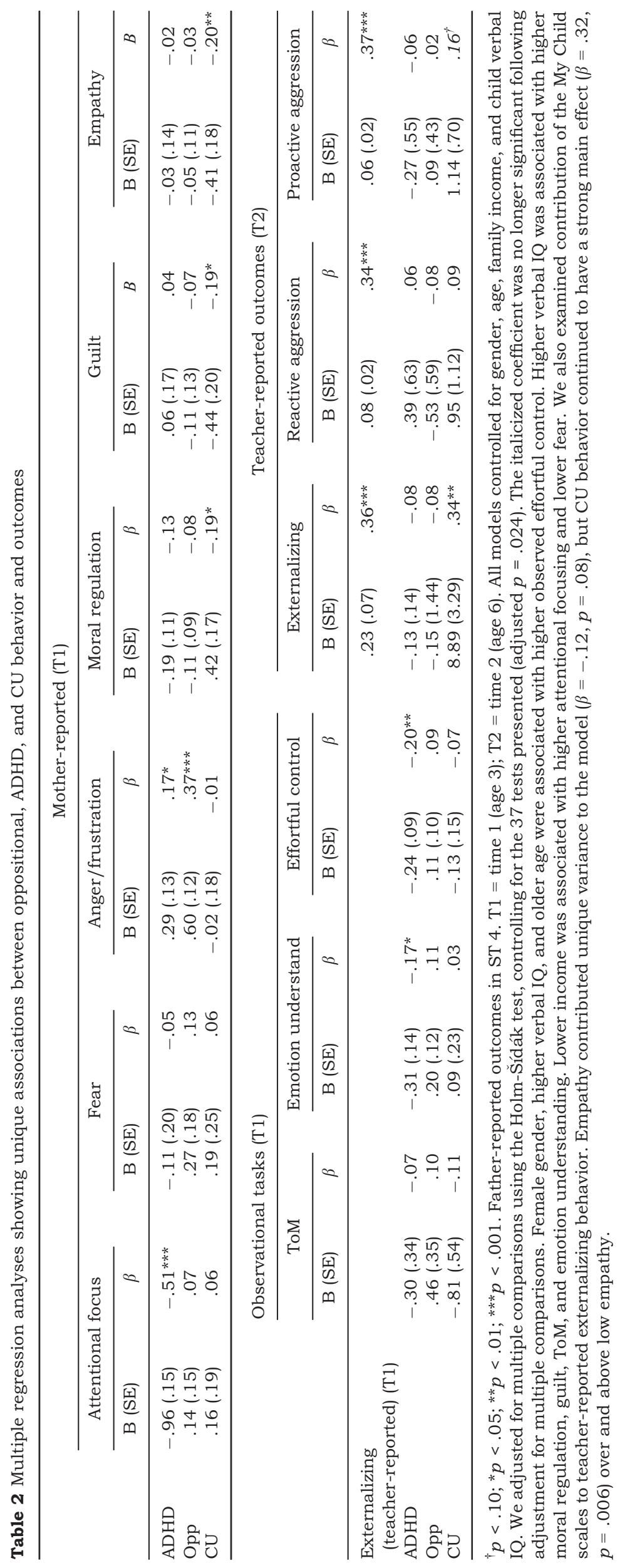




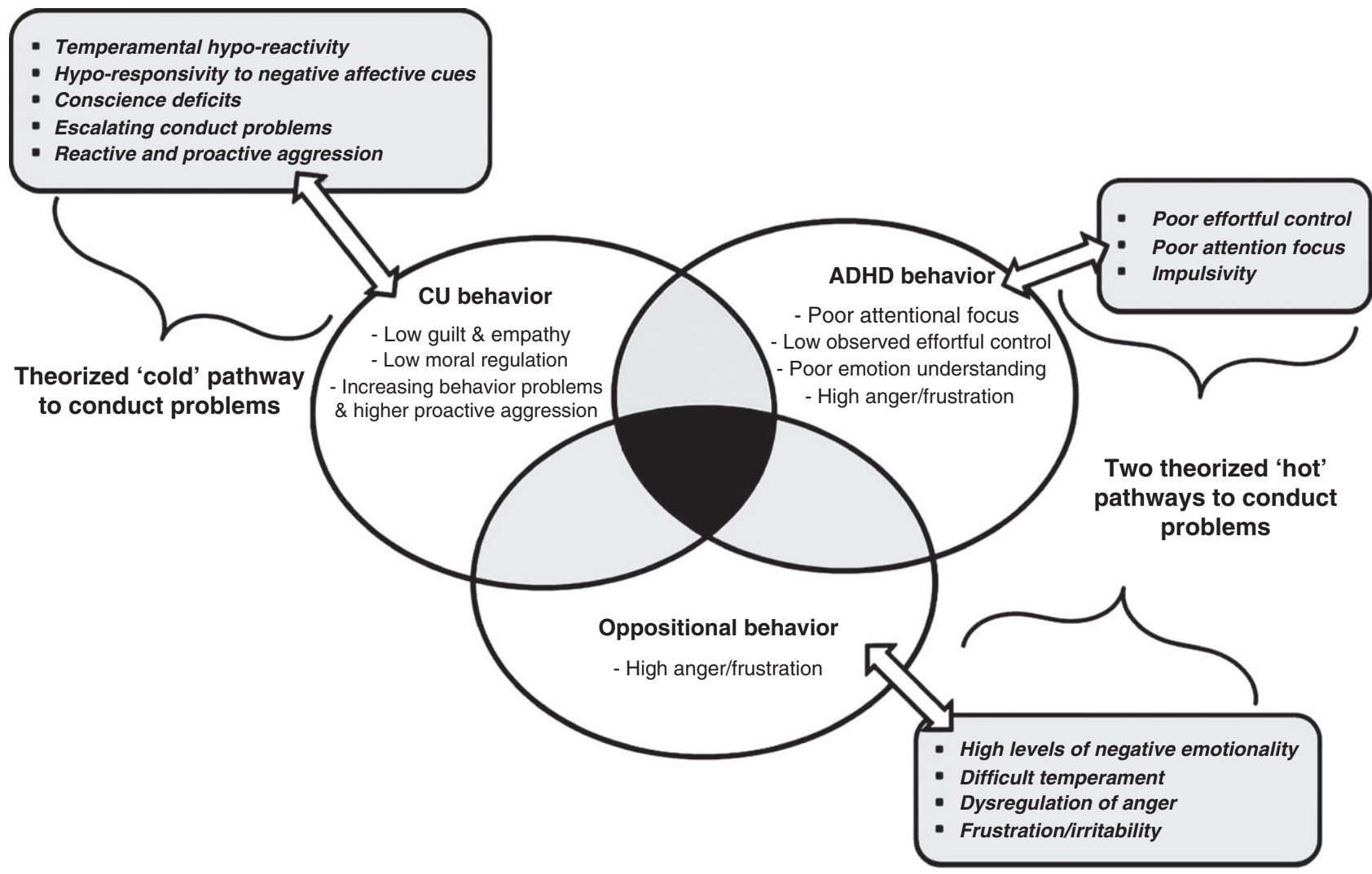

Figure 2 Multiple hypothesized pathways to conduct problems and the unique associations with empirical support in the current paper. Note. The figure presents gray boxes and bold italcized text as hypothesized domains of CP, with 'hot' (ADHD and oppositional) and 'cold' (CU) correlates (Frick \& Morris, 2004; Pardini \& Frick, 2013). White circles represent the three behavior dimensions assessed in this study. Gray/black shading in the circles represents the overlap between CU, oppositional, and ADHD factors. Within circles, we present unique correlates with empirical support in the current study suggesting three distinct nomological networks linked to early-starting CP (gray boxes). Note that in an alternative conceptualization, ODD is proposed to have separate 'headstrong', 'hurtful', and 'irritable' dimensions (see Stringaris \& Goodman, 2009)

behavior. However, many large, longitudinal datasets starting early in development have toddler-aged CBCL data at their disposal. Therefore, demonstrating distinctiveness of CU, ADHD, and oppositional scales, and predictive validity of the $\mathrm{CU}$ behavior scale has merit in terms of providing empirical support for using these brief, albeit ad hoc, scales. Moreover, the fact that the CBCL-generated $\mathrm{CU}$ behavior measure was uniquely correlated with measures associated with its theorized nomological network adds to the justification for its use. Fourth, it is noteworthy that alternative conceptualizations of oppositionality focus on distinct 'irritable', 'headstrong', and 'hurtful' dimensions (Stringaris \& Goodman, 2009). When we reexamined models just using 'irritable' items from our CBCL oppositional scale, the pattern of findings remained unchanged. Nevertheless, future studies are needed to examine how alternative frameworks for conceptualizing oppositional behavior associated with early-starting $\mathrm{CP}$ can be reconciled. Indeed, the proposed 'irritable' versus 'hurtful' dimensions highlight that oppositionality likely comprises both 'hot' and 'cold' elements. Finally, our analytic approach meant that we examined correlates of CU, ADHD, and oppositional behaviors, controlling for their overlap. Thus, while we identified unique associations within variablecentered analyses, future studies are needed to replicate these findings via person-centered analyses. In addition, future studies are needed that assess interactive, reciprocal, and predictive effects of these overlapping domains of early $\mathrm{CP}$ either within variable- or person-centered analyses (see Willoughby et al., 2014).

In conclusion, items from the toddler-aged CBCL differentiated between ADHD, oppositional, and CU behaviors among 3-year olds. In addition, there were unique correlates of these behavior subscales supporting their distinct nomological networks. In particular, ADHD scores were related to lower effortful control; oppositional behavior was related to higher anger/frustration; and $\mathrm{CU}$ behavior was related conscience deficits and predicted higher school-aged CP. Thus, our results support the existence of unique domains of early-starting $\mathrm{CP}$, which has implications for the development of etiologicallybased early intervention strategies. In particular, the current study adds to the growing body of literature highlighting the need for personalized treatments based on specific child characteristics. For example, a focus on salient affective aspects of situations may help promote behavior change in 
children with high CU behavior who may be less able to moderate their behavior in response to the emotional distress of others (for further discussion, see Hyde, Waller, \& Burt, 2014).

\section{Supporting information}

Additional Supporting Information may be found in the online version of this article:

Table s1. Bivariate correlations between mother- and father-reported measures.

Table S2. Descriptive statistics and bivariate correlations between main study variables at T1 and T2.

Table s3. Confirmatory Factor Analysis models for mother-reported CBCL.

Table S4. Synopsis of Confirmatory Factor Analysis models of father-reported 17 CBCL items.

Table S5. Multiple regression analyses showing associations.

Table s6. Multiple regression analyses showing unique associations between combined mother- and father-reported scores.

\section{Acknowledgements}

This research was supported by Grant \#R01MH57489 from the National Institute of Mental Health to Olson. Some findings reported in full here were presented at the International Society for Research in Aggression (ISRA) in Atlanta, GA, USA in July 2014.

The authors thank the children, parents, and teachers who shared their time and many individuals who helped with data collection, particularly Meribeth Gandy Pezda, David Kerr, and Nestor Lopez-Duran. The authors thank administrators of the UM Children's Center as well as the three anonymous reviewers for valuable comments on an earlier version of this article.

The authors have declared that they have no competing or potential conflicts of interest.

\section{Correspondence}

Luke W. Hyde, Department of Psychology, 530 Church Street, University of Michigan, Ann Arbor, MI 48109, USA; Email: LukeHyde@umich.edu

\section{Key points}

- Multiple domains of childhood conduct problems (CP) have been proposed, distinguished by high emotional dysregulation (oppositional behavior), lack of inhibition (ADHD behavior), and emotional hyporesponsivity and conscience deficits (CU behavior).

- In support of this proposal, ADHD behavior was related to lower attentional focus and effortful control, and oppositional behavior to higher anger/frustration, consistent with 'hot' CP.

- CU behavior was related to conscience deficits, consistent with 'cold' CP.

- CU behavior uniquely predicted higher teacher-reported externalizing behavior and more proactive aggression 3 years later.

- Findings support the existence of multiple domains of early-starting CP.

\section{Notes}

1. We found factorial invariance when examining model fit across gender, suggesting the three-factor model fit equally well for boys and girls at age 3 .

2. We reexamined all models using a three-item oppositional behavior scale comprising only the 'irritable' items ('Angry moods', 'Stubborn, sullen, irritable', and 'Temper tantrums / hot temper'). The pattern of findings was unchanged. (cf., distinction between 'irritable' vs. 'headstrong' dimensions of ODD; see Stringaris \& Goodman, 2009).

3. We examined interactions between CU, ADHD, and oppositional behavior and gender in predicting outcomes. One significant interaction emerged between $\mathrm{CU}$ behavior and gender emerged for the model predicting guilt $(p=.097)$. CU behavior predicted lower guilt among females $(\beta=-.36$, $p=.002)$ but not males $(\beta=-.06, p=.666)$. However, 29 interactions were not significant indicating little meaningful interaction among these constructs in this sample.

\section{References}

Achenbach, T.M. (1992). Manual for the child behavior checklist 2/3 and 1992 profile. Burlington: University of Vermont, Department of Psychiatry.

Achenbach, T.M. (1997). Guide for the caregiver-teacher report for ages 2-5. Burlington: University of Vermont, Department of Psychiatry.

Ahadi, S.A., Rothbart, M.K., \& Ye, R. (1993). Children's temperament in the US and China: Similarities and differences. European Journal of Personality, 7, 359-378.

Barkley, R.A. (1997). Behavioral inhibition, sustained attention, and executive functions: Constructing a unifying theory of ADHD. Psychological Bulletin, 121, 65-94.

Baron-Cohen, S., \& Wheelwright, C. (2004). The Empathy Quotient (EQ). An investigation of adults with Asperger Syndrome of high functioning Autism, and normal sex differences. Journal of Autism and Developmental Disorders, 34, 163-175.

Bartsch, K., \& Wellman, H. (1989). Young children's attribution of action to beliefs and desires. Child Development, 60, 946-964.

Blair, R.J.R. (2005). Responding to the emotions of others: Dissociating forms of empathy through the study of typical and psychiatric populations. Consciousness and Cognition, 14, 698-718. 
Blair, R.J.R. (2013). The neurobiology of psychopathic traits in youths. Nature Reviews Neuroscience, 14, 786-799.

Denham, S.A. (1986). Social cognition, prosocial behavior, and emotion in preschoolers: Contextual validation. Child Development, 57, 194-201.

Dodge, K.A., \& Coie, J.D. (1987). Social-information-processing factors in reactive and proactive aggression in children's peer groups. Personality and Social Psychology, 53, 1146-1158.

Frick, P.J., \& Morris, A.S. (2004). Temperament and developmental pathways to conduct problems. Journal of Clinical Child and Adolescent Psychology, 33, 54-68.

Frick, P.J., Ray, J.V., Thornton, L.C., \& Kahn, R.E. (2014). Can callous-unemotional traits enhance the understanding, diagnosis, and treatment of serious conduct problems in children and adolescents? A comprehensive review. Psychological Bulletin, 140, 1-57.

Hu, L.T., \& Bentler, P.M. (1999). Cutoff criteria for fit indexes in covariance structure analysis: Conventional criteria versus new alternatives. Structural Equation Modeling, 6, 1-55.

Hyde, L.W., Shaw, D.S., Gardner, F., Cheong, J., Dishion, T.J., \& Wilson, M. (2013). Dimensions of callousness in early childhood: Links to problem behavior and family intervention effectiveness. Development and Psychopathology, 25, 347-363.

Hyde, L.W., Waller, R., \& Burt, S.A. (2014). Commentary: Improving treatment for youth with callous-unemotional traits through the intersection of basic and applied sciencereflections on Dadds, et al. (2014). Journal of Child Psychology and Psychiatry, 55, 781-783.

Jones, A.P., Happé, F.G., Gilbert, R., Burnett, S., \& Viding, E. (2010). Feeling, caring, knowing: Different types of empathy deficit in boys with psychopathic tendencies and autism spectrum disorder. Journal of Child Psychology and Psychiatry, 51, 1188-1197.

Kazdin, A.E., Whitley, M., \& Marciano, P.L. (2006). Childtherapist and parent-therapist alliance and therapeutic change in the treatment of children referred for oppositional, aggressive, and antisocial behavior. Journal of Child Psychology and Psychiatry, 47, 436-445.

Kerr, D.C., Lopez, N.L., Olson, S.L., \& Sameroff, A.J. (2004). Parental discipline and externalizing behavior problems in early childhood: The roles of moral regulation and child gender. Journal of Abnormal Child Psychology, 32, 369383.

Kochanska, G. (1997). Multiple pathways to conscience for children with different temperaments: From toddlerhood to age 5. Developmental Psychology, 33, 228-240.

Kochanska, G., DeVet, K., Goldman, M., Murray, K., \& Putnam, S.P. (1994). Maternal reports of conscience development and temperament in young children. Child Development, 65, 852-868.

Kochanska, G., Murray, K., Jacques, T.Y., Koenig, A.L., \& Vandegeest, K.A. (1996). Inhibitory control in young children and its role in emerging internalization. Child Development, 67, 490-507.

Lane, J.D., Wellman, H.M., Olson, S.L., LaBounty, J., \& Kerr, D.C.R. (2010). Theory of mind and emotion understanding predict moral development in early childhood. British Journal of Developmental Psychology, 28, 871-889.

Muthén, L.K., \& Muthén, B.O. (1998-2007). Mplus user's guide (5th edn). Los Angeles, CA: Muthén \& Muthén.

Odgers, C.L., Caspi, A., Nagin, D.S., Piquero, A.R., Slutske, W.S., Milne, B.J., . . \& \& Moffitt, T.E. (2008). Is it important to prevent early exposure to drugs and alcohol among adolescents? Psychological Science, 19, 1037-1044.

Olson, S.L., Lopez-Duran, N., Lunkenheimer, E.S., Chang, H., \& Sameroff, A.J. (2011). Individual differences in the development of early peer aggression: Integrating contributions of self-regulation, theory of mind, and parenting. Development and Psychopathology, 23, 253-266.

Olson, S.L., Sameroff, A.J., Kerr, D.C., Lopez, N.L., \& Wellman, H.M. (2005). Developmental foundations of externalizing problems in young children: The role of effortful control. Development and Psychopathology, 17, 25-45.

Pardini, D., \& Frick, P.J. (2013). Multiple developmental pathways to conduct disorder: Current conceptualizations and clinical implications. Journal of the Canadian Academy of Child and Adolescent Psychiatry, 22, 20-25.

Schafer, J.L., \& Graham, J.W. (2002). Missing data: Our view of the state of the art. Psychological Methods, 7, 147-177.

Schmitt, N. (1996). Uses and abuses of coefficient alpha. Psychological Assessment, 8, 350-353.

Shaw, D.S., Gilliom, M., \& Giovannelli, J. (2000). Aggressive behavior disorders. In C.H. Zeanah (Ed.), Handbook of infant mental health (2nd edn). (pp. 397-411). New York: Guilford Press.

Shaw, D.S., \& Gross, H.E. (2008). What we have learned about early childhood and the development of delinquency. In A.K. Liberman (Ed.), The long view of crime: A synthesis of longitudinal research (pp. 79-127). New York: Springer.

von Stauffenberg, C., \& Campbell, S.B. (2007). Predicting the early developmental course of symptoms of attention deficit hyperactivity disorder. Journal of Applied Developmental Psychology, 28, 536-552.

Stringaris, A., \& Goodman, R. (2009). Three dimensions of oppositionality in youth. Journal of Child Psychology and Psychiatry, 50, 216-223.

Wechsler, D. (1989). WPPSI-R, manual: Wechsler preschool and primary scale of intelligence, revised. New York, NY: Psychological Corporation.

Willoughby, M.T., Mills-Koonce, W.R., Gottfredson, N.C., \& Wagner, N.J. (2014). Measuring callous unemotional behaviors in early childhood: Factor structure and the prediction of stable aggression in middle childhood. Journal of Psychopathology and Behavioral Assessment, 36, 30-42. Erratum in Journal of Psychopathology and Behavioral Assessment, 36, 43-46

Willoughby, M.T., Waschbusch, D.A., Moore, G.A., \& Propper, C.B. (2011). Using the ASEBA to screen for callous unemotional traits in early childhood: Factor structure, temporal stability, and utility. Journal of Psychopathology and Behavioral Assessment, 33, 19-30.

Accepted for publication: 26 August 2014 